\title{
An adaptive resolution full-waveform inversion using a mesh-free finite-difference method
}

\author{
Junichi TAKEKAWA $^{1}$ and Hitoshi MIKADA ${ }^{1}$ \\ ${ }^{1}$ Dept. of Civil and Earth Res. Eng., Kyoto University
}

\begin{abstract}
In the present study, we propose a new strategy of full-waveform inversion with adaptive resolution for reducing the computational costs (calculation time, computational memory). We adopt a mesh-free finite difference method to calculate wavefield and waveforms at receivers. Since the method can carry out the forward modeling without regular lattice or mesh structure, an adaptive refinement of calculation points could be applied to complex velocity structures in a simple manner. We introduce the outline of the proposed approach, and demonstrate the effectiveness of our method using a numerical experiment. Our results show the adaptive refinement of the calculation points only around low velocity zones. This indicates that our approach can reduce the computational burden in simple and automatic manners.
\end{abstract}

\section{INTRODUCTION}

Recently, the full-waveform inversion (FWI) has been widely used for inverting the velocity structure in the sub-surface. FWI extracts quantitative information from seismograms based on full-wavefield modeling. Since high-resolution imaging at half the propagated wavelength is expected, many researchers and engineers focus attention on the development of $\mathrm{FWI}^{1}$. One of the key factors of FWI is an efficient full-wavefield simulator because the accuracy of the estimated gradient or Hessian is highly dependent on the accuracy of it.

The finite difference methods (FDMs) have been used as simulators for the full-wavefield modeling. Although FDM can provide accurate solutions with simple calculation procedures, the coefficients of operators are built based on the regular lattice grid structure. This complicates an efficient arrangement of calculation grids which adapts to an arbitrary velocity structure. Since the heavy computational burden is the weak point of FWI, new methods which reduce the computational costs have been waited for.

In the present study, we apply a mesh-free method $^{2)}$ as a full-wavefield simulator. The method can provide full-wavefield without mesh or regular lattice. This means that an adaptive resolution of calculation points corresponding to velocity structure in the subsurface can be achieved in a simple manner. We apply the mesh-free method to FWI. We conduct a numerical experiment to demonstrate the effectiveness of our approach.

\section{METHOD}

We briefly explain the mesh-free method and its application strategy to FWI.

\section{(1) Mesh-free FD method}

The mesh-free concept is based on the multi-variable Taylor expansion. The multi-variable Taylor expansion of a scalar function $f(r)$ to M-th order at position $\mathbf{r}_{\mathbf{i}}$ is expressed as follows:

$\mathrm{f}\left(\mathbf{r}_{\mathrm{i}}+\Delta \mathbf{r}\right)=\mathrm{f}\left(\mathbf{r}_{\mathrm{i}}\right)+\sum_{\mathrm{m}=1}^{\mathrm{M}}\left\{\frac{1}{\mathrm{~m} !}(\Delta \mathbf{r} \cdot \nabla)^{\mathrm{m}_{\mathrm{f}}} \mathrm{f}\left(\mathbf{r}_{\mathrm{i}}\right)\right\}+$
$\mathrm{O}\left(\|\Delta \mathbf{r}\|^{\mathrm{M}+1}\right)$

where $\mathbf{r}_{\mathrm{i}}$ and $\mathbf{r}_{\mathbf{i}}+\Delta \mathbf{r}$ are the position vectors of grid $i$ and its neighboring grid $j$, respectively. In two-dimensional case, differential operators are calculated as follows:

$$
\begin{aligned}
& (\boldsymbol{\delta})_{\mathrm{r}=\mathrm{r}_{\mathrm{i}}} \approx\left\{\sum_{\mathrm{j}=1}^{\mathrm{n}}(\mathbf{P} \otimes \mathbf{P})\right\}^{-1} \cdot\left\{\sum_{\mathrm{j}=1}^{\mathrm{n}}\left(\mathbf{P} \Delta \mathrm{f}_{\mathrm{ij}}\right)\right\} \\
& \mathbf{P}=\left(\Delta \mathrm{r}_{1}, \Delta \mathrm{r}_{2}, \frac{1}{2 !} \Delta \mathrm{r}_{1}^{2}, \Delta \mathrm{r}_{1} \Delta \mathrm{r}_{2}, \frac{1}{2 !} \Delta \mathrm{r}_{2}^{2}, \cdots, \frac{1}{\mathrm{M} !} \Delta \mathrm{r}_{2}^{\mathrm{M}}\right)^{\mathrm{T}} \\
& \boldsymbol{\delta}=\left(\frac{\partial}{\partial \mathrm{r}_{1}}, \frac{\partial}{\partial \mathrm{r}_{2}}, \frac{\partial^{2}}{\partial \mathrm{r}_{1}^{2}}, \frac{\partial^{2}}{\partial \mathrm{r}_{1} \partial \mathrm{r}_{2}}, \frac{\partial^{2}}{\partial \mathrm{r}_{2}^{2}}, \cdots, \frac{\partial^{\mathrm{M}}}{\partial \mathrm{r}_{2}^{\mathrm{M}}}\right)^{\mathrm{T}}
\end{aligned}
$$

where $f_{i j}=f_{j}-f_{i}$ is the difference between scalar values at positions $\mathbf{r}_{\mathrm{i}}$ and $\mathbf{r}_{\mathrm{j}}$. The details of the derivation of the above equations can be found in a reference ${ }^{2}$.

\section{(2) FWI with adaptive resolution}

The velocity is determined by minimizing the 
misfit function $\mathrm{E}$ as follows;

$$
\mathrm{E}=\frac{1}{2} \sum_{\mathrm{s}} \sum_{\mathrm{r}} \int \mathrm{dt}\left\{\mathrm{P}_{\text {cal }}\left(\mathbf{x}_{\mathrm{r}}, \mathrm{t}\right)-\mathrm{P}_{\mathrm{obs}}\left(\mathbf{x}_{\mathrm{r}}, \mathrm{t}\right)\right\}^{2}
$$

where $\mathrm{P}_{\text {obs }}$ is the observed data and $\mathrm{P}_{\text {cal }}$ is the calculated data using the current velocity model. E is minimized by the gradient or steepest-descent direction $^{3)}$ as follows;

$\gamma_{\mathrm{n}}=-\frac{1}{\mathrm{~V}_{\mathrm{n}}^{3}} \sum_{\mathrm{s}} \int \mathrm{dt}\left(\partial_{\mathrm{t}} \mathrm{P}_{\mathrm{f}}\right)\left(\partial_{\mathrm{t}} \mathrm{P}_{\mathrm{b}}\right)$

where $\mathrm{P}_{\mathrm{f}}$ and $\mathrm{P}_{\mathrm{b}}$ are the forward and backward reverse-time wave propagation, respectively, $\mathrm{n}$ is iteration step number. The velocity field is updated as follows;

$\mathrm{V}_{\mathrm{n}+1}=\mathrm{V}_{\mathrm{n}}+\alpha \gamma_{\mathrm{n}}$

where $\alpha$ is a step length which is estimated by a line search method.

\section{NUMERICAL RESULTS}

In this chapter, we, first, present the effectiveness of the mesh-free FD method. We, then, apply the mesh-free FD method to FWI with adaptive resolution.

\section{(1) Mesh-free FD method}

Figure 1 shows an example of the acoustic wave propagation using the mesh-free FD method ${ }^{2}$. The method enables us to simulate wave propagation using irregular arrangement of calculation points. In the method, re-meshing process required in finite element methods is not needed. This indicates that arbitrary refinement of spatial resolution can be achieved in a simple manner.

In FWI, the velocity model changes step by step. In general, the near surface has lower velocity than the deeper part. We can use the discontinuous grid $^{4)}$ for efficient calculation. Recently, more complex velocity structures become the target of seismic exploration such as BP model, SEG-EAGE model, etc. In this case, the discontinuous grid does not (a)

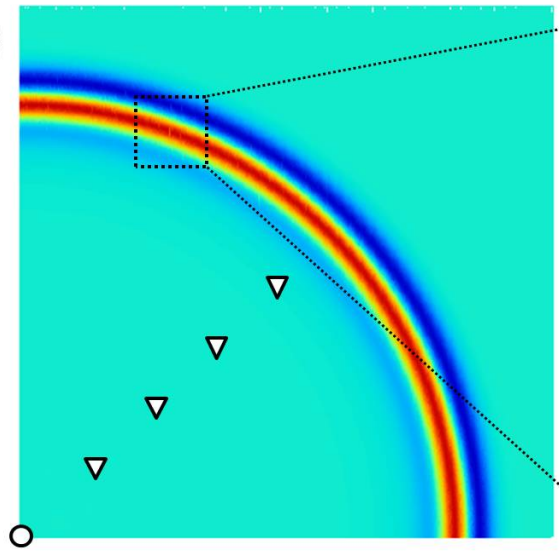

(b)

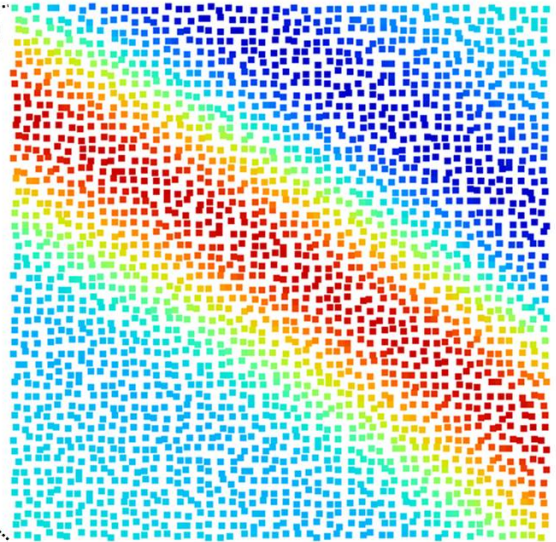

Figure 1: (a) Snapshot of acoustic wave propagation. (b) Close-up figure in dotted square in (a). The figures are modified from Takekawa et al. $(2015)^{2}$.

Velocity structure

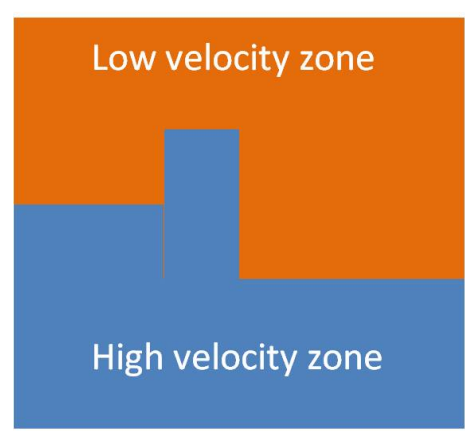

Nodal distribution

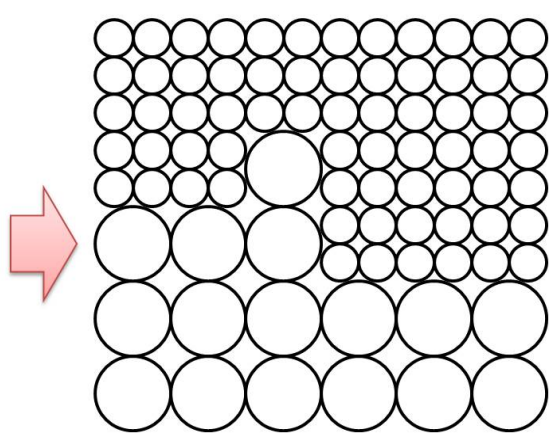

Figure 2: Schematic figure of discretization in the mesh-free FD method. 


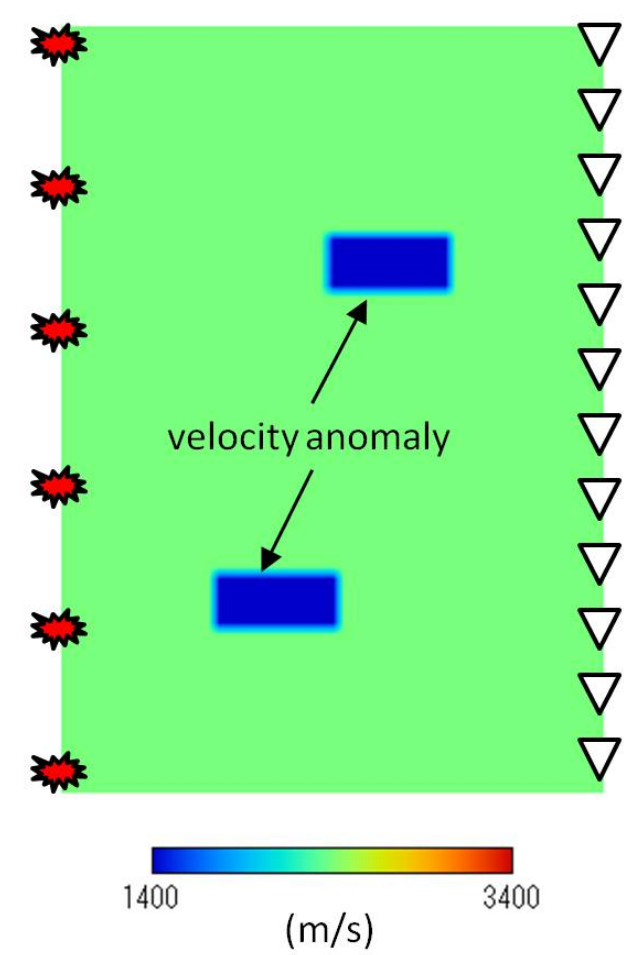

Figure 3: Velocity structure of a numerical experiment. The model includes two low velocity anomalies. 16 acoustic sources and 56 receivers are set to left and right edges of the model, respectively.

work well enough to be used for efficient calculation. However, the mesh-free FD method can use adaptive arrangement of calculation points due to its mesh-free concept. The schematic figure is shown in Figure 2. This is the idea behind the application of the mesh-free FD method to FWI.

\section{(2) Adaptive resolution FWI}

We demonstrate the effectiveness of our approach using a simple model as shown in Figure 3. The model has two low velocity anomalies whose velocity is $1400 \mathrm{~m} / \mathrm{s}$. The number of sources and receivers are 16 and 56, respectively. The initial velocity model of FWI has a uniform velocity of $2400 \mathrm{~m} / \mathrm{s}$.

Figure 4 shows the result of FWI using the mesh-free FD method. We show not only the inverted velocity structure but also the distribution of calculation points. In the inverted velocity, two low velocity zones start to appear at the third iteration. At the eighth iteration, low velocity anomalies are inverted clearly. On the other hand, right side of the figure shows the distribution of calculation points. At the first iteration, the arrangement of the points is uniform because the velocity structure is almost homogeneous. At the third iteration, on the other hand, fine distribution of the points can be observed. This zone
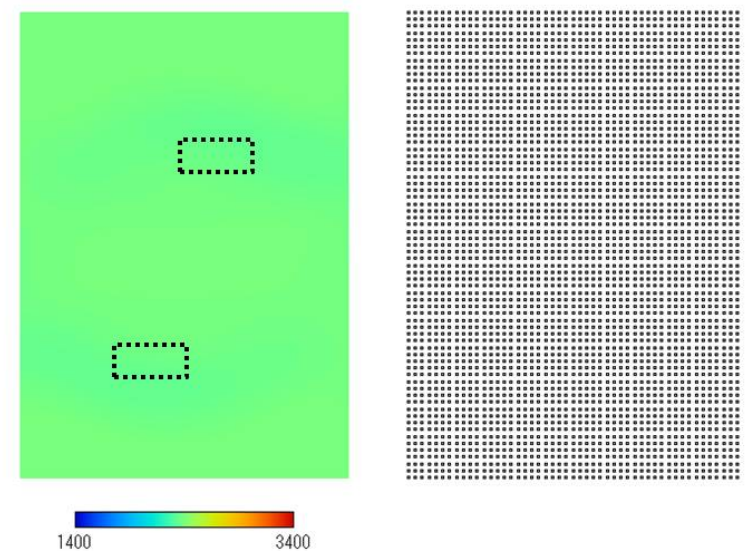

(a) first iteration
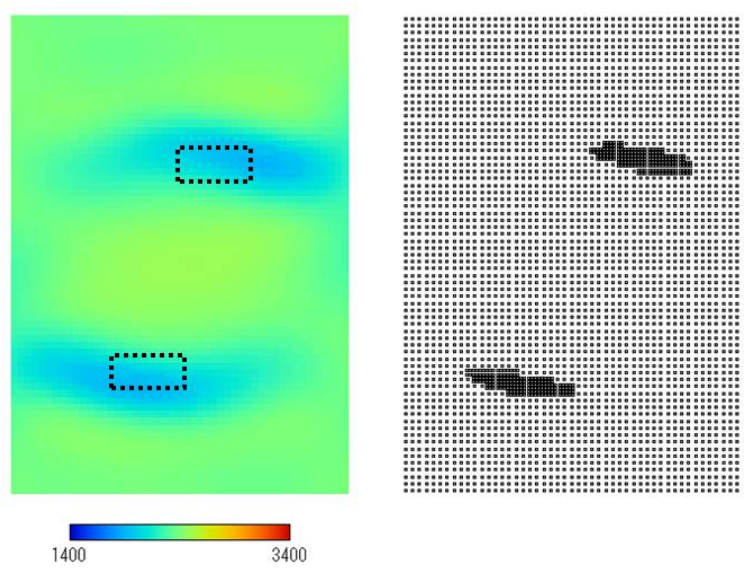

(b) third iteration
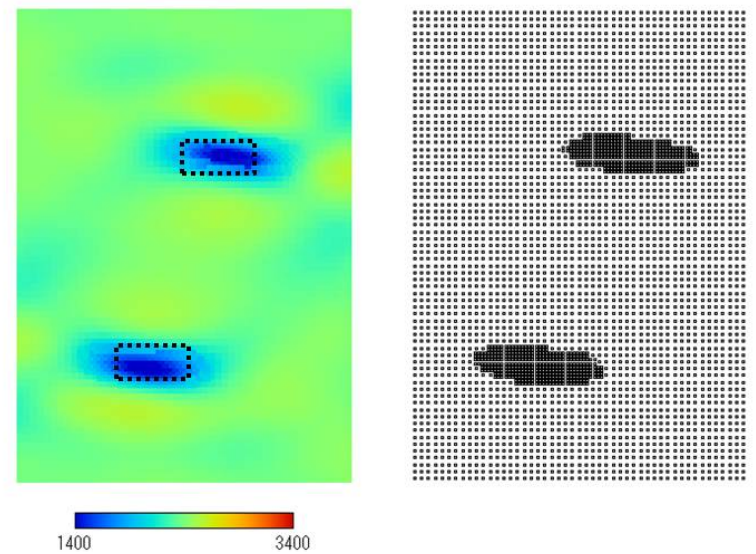

(c) eighth iteration

Figure 4: Inversion results of the adaptive resolution FWI with the mesh-free FD method. Left and right figures are inverted velocity structures and distributions of calculation points, respectively. In the right figures, black dots represent the calculation points.

corresponds to low velocity zone in the left figure. In eighth iteration, spreading of finer region can be observed. At the low velocity zone, finer calculation points are needed to avoid numerical dispersion. 


\section{CONCLUSIONS}

We proposed a new strategy to reduce computational burden of FWI using a mesh-free FD method. The mesh-free FD method can treat arbitrary shaped target in a simple manner. This means that the method does not require cumbersome procedures such as re-meshing in FEM. The refinement of the spatial resolution is conducted just by setting additional calculation points.

We conducted a numerical experiment to validate the effectiveness of our strategy. The result shows that the adaptive refinement of the spatial resolution can be achieved in a simple manner. This indicates that our strategy works well in treating complex velocity structures.

In the future work, we will expand our strategy to three-dimensional problems whose computational burden becomes the critical issue.

ACKNOWLEDGMENT: This work was partly supported by MEXT/JSPS KAKENHI Grant Number 15K18301.

\section{REFERENCES}

1) Virieux, J. and S. Operto, 2009, An overview of full-waveform inversion in exploration geophysics, Geophysics, 74, 6, WCC127-WCC152.

2) Takekawa, J., H. Mikada, and N. Imamura, 2015, A mesh-free method with arbitrary-order accuracy for acoustic wave propagation, Computers \& Geosciences, 78, 15-25.

3) Tarantola, A., 1984, Inversion of seismic reflection data in the acoustic approximation, Geophysics, 49, 1259-1266.

4) Aoi, S. and H. Fujiwara, 1999, 3D finite-difference method using discontinuous grids, Bulletin of the Seismological Society of America, 89, 4, 918-930. 